\title{
Correction to: J Interferon Cytokine Res 32(8):368-377
}

$\mathrm{I}^{\mathrm{N}}$

N THE August 2012 issue of Journal of Interferon \& Cytokine Research (vol. 32, no. 8, pp. 368-377), in the article entitled "Inhibition of Type I Interferon-Mediated Antiviral Action in Human Glioma Cells by the IKK Inhibitors BMS345541 and TPCA-1,' by Dr. Ziyun Du and others, the authors have found errors in both the text and in Figure 3A.

In the Materials and Methods section page 370, it reads "A minimum of 2 independent experiments were performed at least in duplicate. Statistical analysis was performed by Student's $t$-test." This should be "For statistical analysis of the data a minimum of two independent experiments was performed at least in duplicate, and analysis was performed by Student's $t$-test."

In the legend to Figure 3A on page 372, it reads "Cell lysates were prepared from U87 cells pretreated with BMS-345541 $(5 \mu \mathrm{M}$ for $2 \mathrm{~h})$ and then incubated with IFN (1000 U/mL for $1 \mathrm{~h})$, separated by sodium dodecyl sulfate-polyacrylamide gel electrophoresis, and immunoblotted with the indicated antibodies." This should be "Cell lysates were prepared from U87 cells pretreated with BMS-345541 (5 $\mathrm{M}$ for $2 \mathrm{~h})$ and then incubated with IFN (1000 U/mL for $1 \mathrm{~h})$, separated by sodium dodecyl sulfate-polyacrylamide gel electrophoresis, and immunoblotted with the indicated antibodies. The results of one experiment in U87 cells is shown, which is representative of the results obtained in other glioma cell lines (MT330 and GBM6 cells).',

Several mistakes were made in assembling Figure 3A and a corrected Figure 3A is now provided.

These changes do not affect the results or conclusions presented by the authors. The online version of the article has been corrected to reflect these changes.

The authors wish to apologize for the errors.

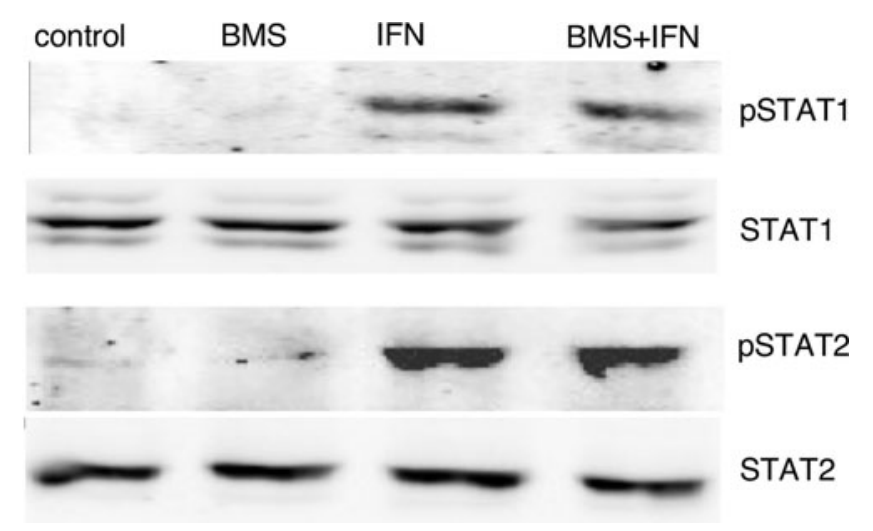

